\title{
Flow friction on co-rotating parallel-disk assemblies with forced through-flow
}

\author{
S. Mochizuki \\ Department of Mechanical Engineering, Tokyo University of Agriculture and Technology, Koganei, Tokyo, Japan \\ Wen-Jei Yang \\ Department of Mechanical Engineering and Applied Mechanics, University of Michigan, Ann Arbor, Michigan 48109, USA
}

\begin{abstract}
The power requirement for a rotating parallel-disk assembly with through-flow is experimentally studied. The flow velocity at the test core exit is measured using a traverse hot-wire probe, from which the exit kinetic energy is determined. A traverse pitot tube is utilized to monitor the velocity distribution in the inlet duct from which the flow rate is evaluated. The total pressure characteristics, fluid power transmission, rotor efficiency and moment coefficient (i.e. power number) are determined from the measurements of static pressures, rotational speed and torque. It is disclosed that the onset of stall propagation, a unique characteristics of shear pump, can be detected from a total pressure plot.
\end{abstract}

\section{Introduction}

The disks in rotating machinery such as turbines of rotary compressors mostly revolve in housings. The disk in a housing is the common idealized model which has been employed to determine the basic performance characteristics of such a rotating device. Owens (1984) presented a review of fluid flows and heat transfer in the rotating disk systems that are relevant to the designs of turbo-machinery. The simplest model is the free disk, consisting of a single disk rotating in an infinite fluid. The flow in the neighborhood of a rotating disk is often used as a reference for comparison with rotary machines. Schlichting (1972) presented a comprehensive analysis of the torques on a free disk and an enclosed disk (the disk in a housing). In both the free and enclosed disks, the flow is characterized by the rotational Reynolds number $R e_{w}$ defined as

$R e_{w}=\frac{u_{2} r_{2}}{v}=\frac{r_{2}^{2} \Omega}{v}$.

Here $r_{2}$ denotes the outer radius of the disk; $\Omega$, angular velocity; and $v$, kinematic viscosity. $u_{2}$ is the tangential velocity at the outer radius. The critical Reynolds number for the transition from laminar to turbulent flow is about $3 \times 10^{5}$, same as the flow about a plate (Schlichting 1972). It is noteworthy that apart from the case of very small gaps, the moment of viscous forces is completely indepen- dent of the width of the gap, $s$. However, the moment on a free disk is greater than that on an enclosed disk.

The present study concerns with fluid flow and torque in the rotor system which consists of a rotating paralleldisk assembly with a single influx, as illustrated in Fig. 1. $d_{1}$ and $d_{2}$ are the inner and outer diameter of the disk, respectively. The power requirement will be experimentally determined and compared with that for a free disk. A series of experimental and theoretical studies (Mochizuki \& Tanida 1979; Mochizuki et al. 1983; Mochizuki \& Yang 1985; Sim \& Yang 1984; Sim \& Yang 1985) have been conducted to determine flow mechanisms and heat transfer in both stationary and rotating parallel-disk assemblies. A stall propagation phenomenon was disclosed in a co-rotating disk assembly when the through-flow rate was low (Mochizuki \& Tanida 1979). It was an extremely unsteady flow with regular periodicity exiting from the disk dim.

Figure 2 is a conceptual illustration of the flow situation in and around the rotor when three rotating stall cells occur. The inner and outer circles indicate respectively the inner and outer rims of the rotor in a clockwise rotation at

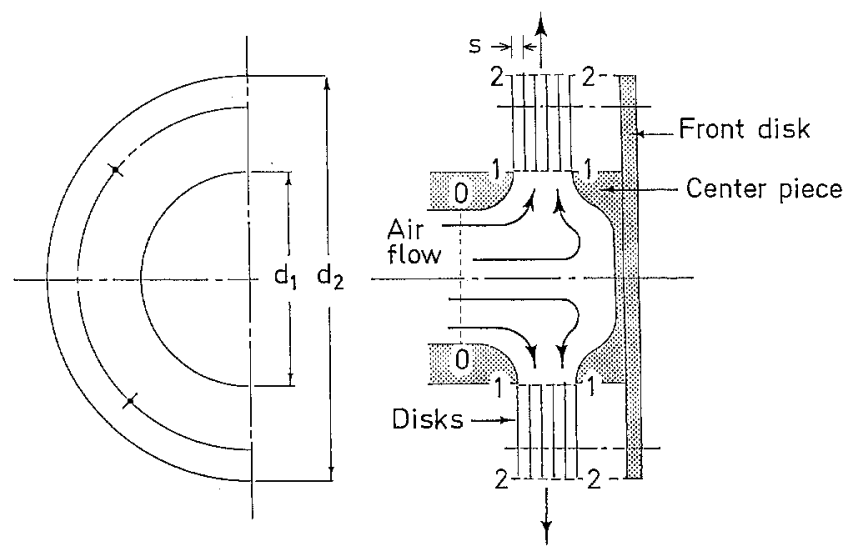

Fig. 1. Schematic of test core: 1 air flow; 2 front disk; 3 center piece; 4 disks 


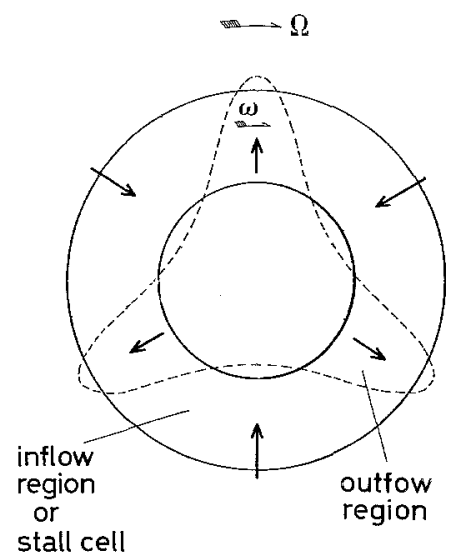

Fig. 2. A conceptual illustration of flow situation at occurrence of three stall cells: 1 inflow region or stall cell; 2 outflow region

an angular velocity $\Omega$. The broken line defines the region in which the flow is radially outward. The flow in the space outside the broken line is radially inward and is called stall cells. The three stall cells are equally spaced circumferentially and rotates in the same direction as the rotor but at a slower angular velocity $\omega$. The amplitude of stall cells increases with a decrease in the through-flow rate. The effects of flow conditions and rotor geometry on the number, rotational speed and pulsation frequency of stall cells were investigated by Mochizuki and Tanida (1979).

The present work represents the first study on the power requirement and fan efficiency of rotating paralleldisk assemblies. A method is developed to predict the onset of stall propagation.

\section{Experimental apparatus and data reduction}

Figure 3 illustrates the test apparatus consisting of a wind tunnel and a rotating parallel-disk assembly. Air entered

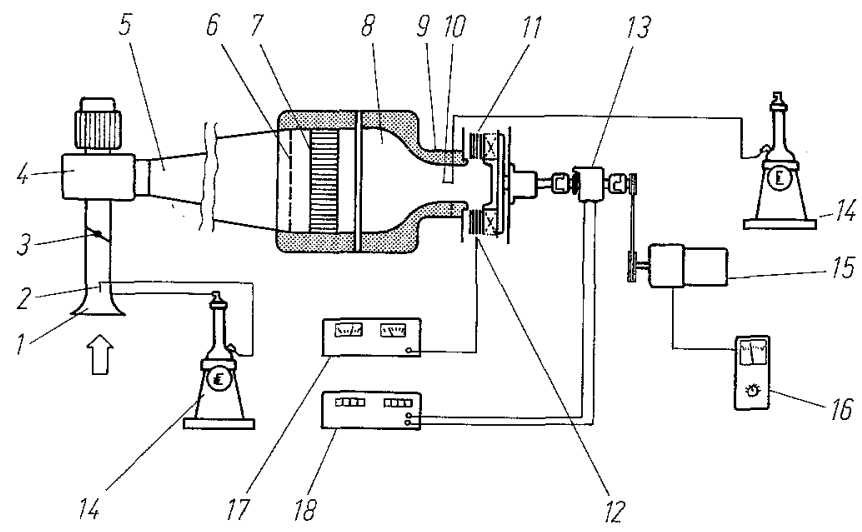

Fig. 3. Schematic of test setup: 1 bell mouth; 2 Pitot tube; 3 damper; 4 turbofan; 5 diffuser; 6 screen; 7 honeycomb; 8 calming chamber; 9 duct; 10 static tube; 11 test core; 12 hot wire probe; $13 \mathrm{rpm}$ and torque detector; 14 manometer; 15 motor; $16 \mathrm{rpm}$ controller; 17 hot wire anemometer and linearizer; 18 torque meter

through a bellmouth and progressed radially into the spaces between the disks. It was accelerated by shear forces and discharged from the rotor (disk assembly) into the surroundings. The damper was installed upstream from the turbofan to regulate the rate of forced air supply. Air was supplied to the rotor from the turbofan through a calming chamber. The rotating speed of the rotor $n$ in revolutions per second was adjusted by a variable-speed motor through the use of a rpm controller. A strain-gauge type torque detector was installed on a shaft between the motor and the rotor. The torque $T_{t}$ monitored by the detector was partially expended as the loss $T_{l}$ which consisted of the friction loss at the bearings and the windage loss at the side surfaces of the disk core. The remaining portion $T$ was then transferred to the fluid flowing through the disk spacings inside the core:

$T=T_{t}-T_{t}$.

Prior to the experiments, $T_{l}$ was measured at various rotational speeds using a test core with the entrance and exit of all disk spacings sealed by masking tapes to prevent the occurrence of a through-flow. Inside the torque detector, a gear was fit on a rotationary shaft in a perpendicular direction with a stationary magnetic field. $n$ was monitored using a rotational (rpm) meter which counted the number of electrical pulses generated when the gear cut the magnetic field. The torque and the power supplied to the fluid flowing through each disk spacing within the core were calculated by the equation

$T^{\prime}=T /(N-1)$ and $L^{\prime} \Omega T$,

respectively.

Here, $N$ denotes the number of disks and $\Omega$ is the angular velocity, equal to $2 \pi n$.

A traverse pitot tube measured the air velocity distribution in a suction duct downstream from the bellmouth. The volumetric through-flow rate $\dot{V}$ was calculated by integrating the distribution across the cross section. The relationship between the midstream velocity and $\dot{V}$ was established prior to the experiments. Thereafter, $\dot{V}$ was read from the calibration curve with the velocity measurement. The volumetric flow rate through each disk spacing was

$\dot{V}^{\prime}=\dot{V} /(N-1)$.

The static pressure rise across the rotor $\Delta P_{s}$ was measured between a static pressure probe located upstream from the rotor (subscript 0 ) and the ambient (subscript 2). The flow velocity at the core inlet $u_{0}$ was

$u_{0}=\dot{V} / A_{0}$,

where $A_{0}$ represents the flow cross-sectional area at the exit from the inlet duct.

Figure 4 shows the positioning of a traverse hot-wire probe at the core exit together with its measured exit 


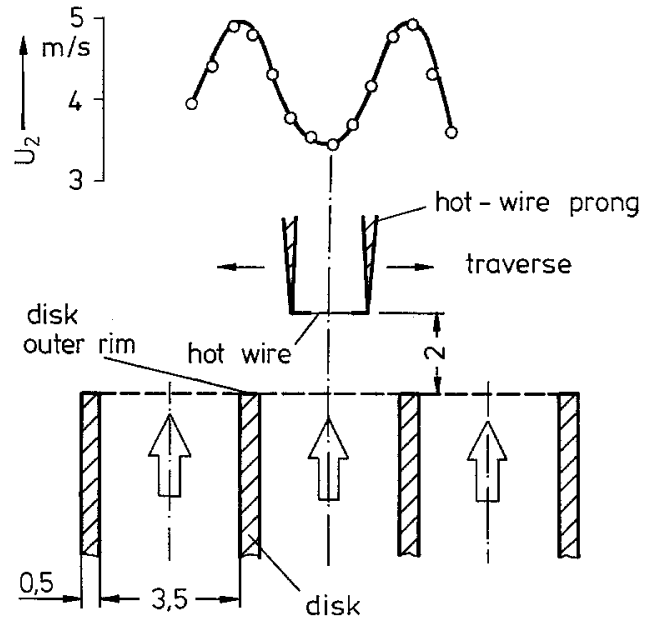

Fig. 4. Position of a traverse hot-wire probe at core exit and its measured exit velocity; $n=600 \mathrm{rpm} ; \dot{V}=4.97 \cdot 10^{-3} \mathrm{~m}^{3} / \mathrm{s}$

velocity. Since $u_{2}$ varied periodically in the direction parallel to the rotating axis, an integrated mean value was employed in the evaluation of the kinetic energy for the total pressure at the core exit. The total pressure rise across the test core is

$\Delta P_{t}=\Delta P_{s}+\varrho\left(u_{2}^{2}-u_{0}^{2}\right) / 2$

in which $\varrho$ signifies the fluid density.

The fan efficiency of the rotor core $\eta$ is defined as the ratio of the mechanical energy increase in the fluid and the power supplied to the fluid from the rotor:

$\eta=\dot{V}^{\prime} \Delta P_{t} / L^{\prime}$.

The dimensionless moment coefficient $C_{m}$ is defined as

$C_{m}=T^{\prime} /\left(1 / 2 \cdot \varrho \Omega^{2} r_{2}^{5}\right)$

for both sides in a disk spacing or a disk wetted on both sides.

\section{Results and discussion}

The test core consisted of multiple disks with opening in the center, as shown in Fig. 1. All disks were made of aluminum $0.5 \mathrm{~mm}$ in thickness with $160 \mathrm{~mm}$ i.d. Both the outer diameter and spacing ( $s$ ) of the disks may be varied to produce different values of the flow length to the hydraulic diameter ratio. However, the present study reports the test results for a core with $d_{2}=345 \mathrm{~mm}$, $s=3.5 \mathrm{~mm}$ and $N=9$.

The characteristics of the total pressure rise across the rotor core $A P_{t}$ is presented in Fig. 5. At low flow rate $\dot{V}^{\prime}$ and high rotational speed $n, \Delta P_{t}$ is positive. It means that the rotor core functions as a fan. On the contrary, $\Delta P_{t}$ is negative for large values of $\dot{V}^{\prime}$, implying that the core becomes a resistance to the through-flow. In the positive
$\Delta P_{t}$ region, $\Delta P_{t}$ exhibits the characteristics of monotonous reduction in its magnitude with an increase in $\dot{V}^{\prime}$. Therefore, one expects a stable operation of the rotor core without surging phenomenon.

A hot-wire probe was placed at the disk periphery. Figure 6 is a typical recording of the signals from the probe at various through-flow rates $\dot{V}^{\prime}$. It indicates that, at a very low flow rate, e.g., $\dot{V}^{\prime}=0.00906 \mathrm{~m}^{3} / \mathrm{s}$, the flow variation had a regular periodicity and a strong stall phenomenon occurred. With an increase in the flow rate, the period of flow fluctuations became shorter, and the

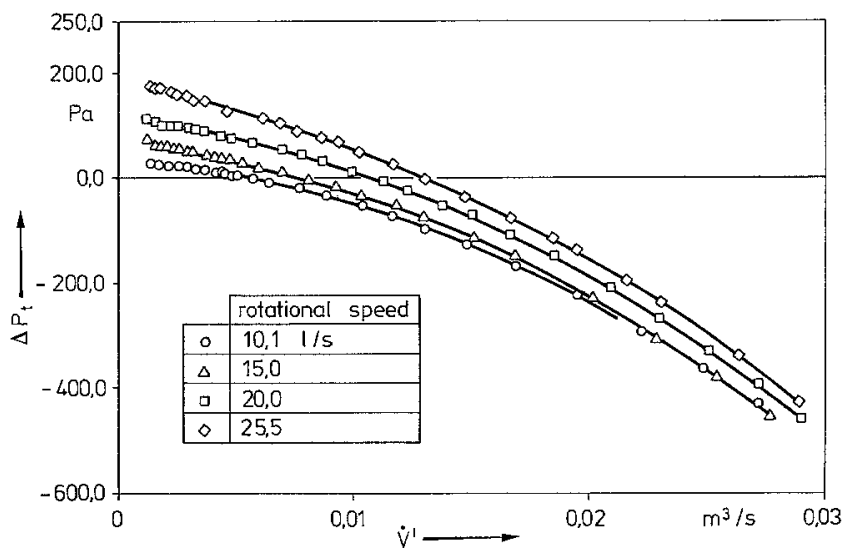

Fig. 5. Total pressure rise across test core versus flow rate
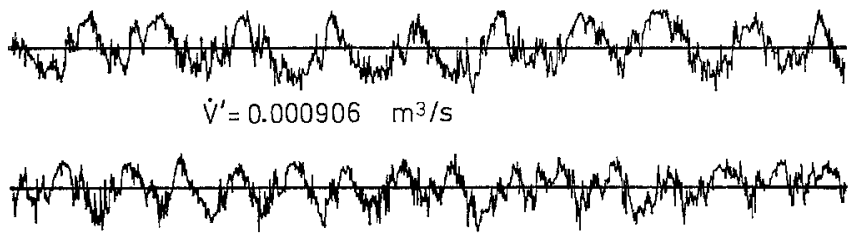
0.00207

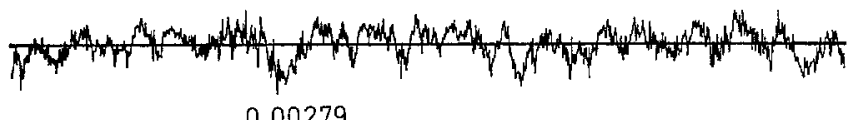

0.00279

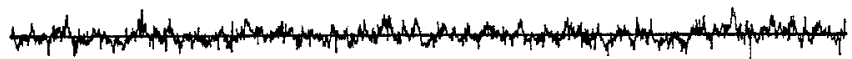
0.00881
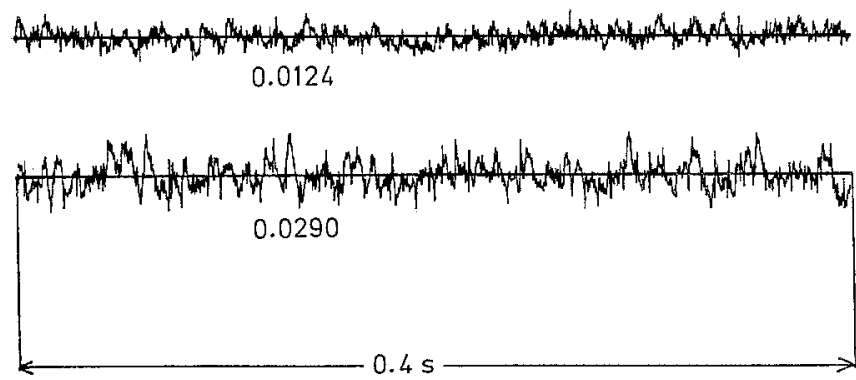

Fig. 6. A typical oscillographic recording of flow fluctuations at the exit from a test core with $d_{1}=160 \mathrm{~mm}, d_{2}=345 \mathrm{~mm}$, $s=3.5 \mathrm{~mm}$ and $n=20 \mathrm{~Hz}$ 


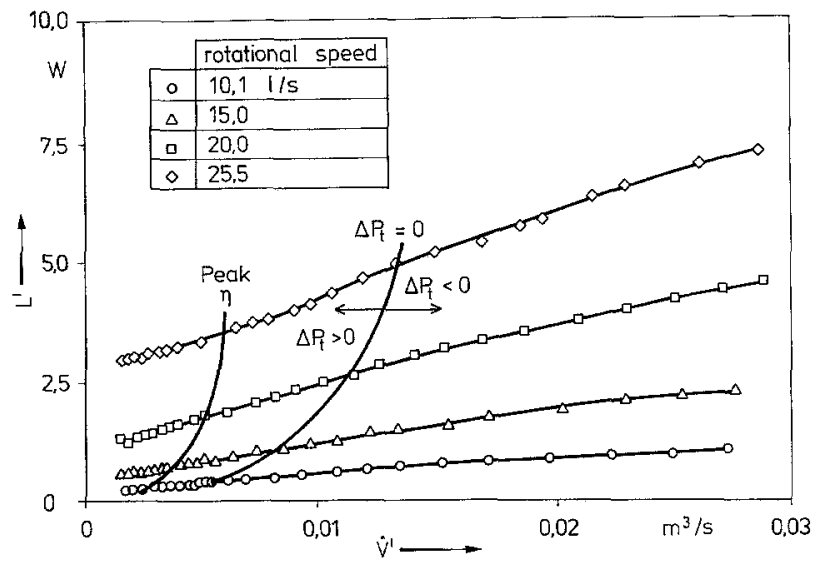

Fig. 7. Fluid power consumption versus flow rate

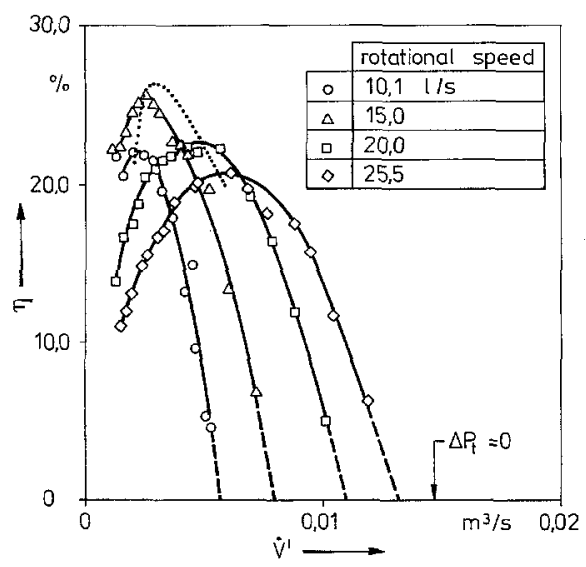

Fig. 8. Fan efficiency versus flow rate

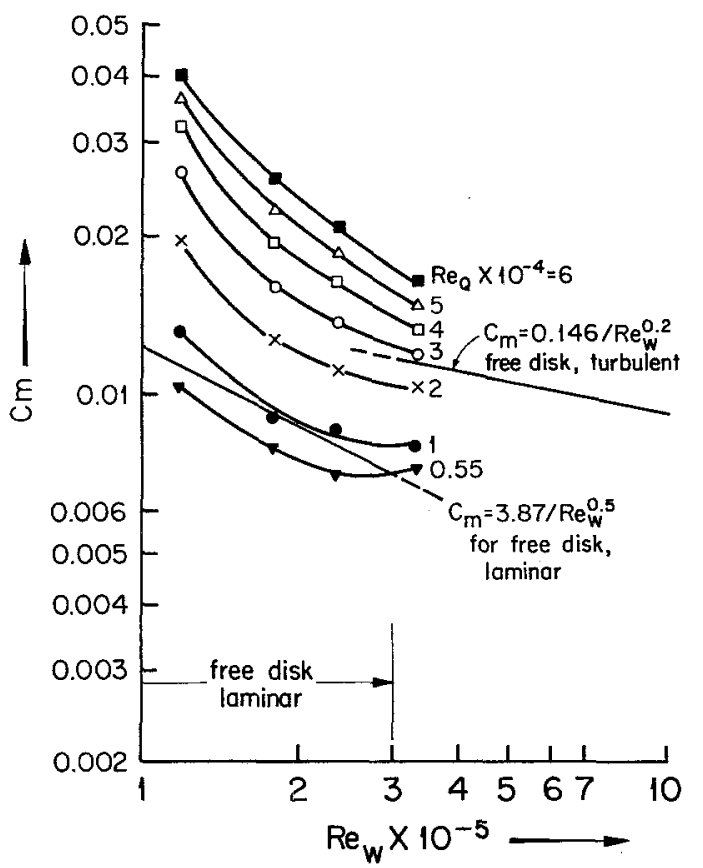

Fig. 9. Dimensionless moment coefficient versus rotational Reynolds number amplitude reduced because of an increase in the number of stall cells. The fluctuation frequency became minimum and the stall phenomenon ceased at a flow rate between $0.00881 \mathrm{~m}^{3} / \mathrm{s}$ and $0.0124 \mathrm{~m}^{3} / \mathrm{s}$. With a further increase in $\dot{V}^{\prime}$, the flow fluctuating amplitude began to increase but no more periodicity existed in the flow fluctuation. It exhibited a random oscillation, typical in turbulent flow. One may thus conclude that the stall phenomenon vanishes at $\dot{V}^{\prime}$ of about $0.01 \mathrm{~m}^{3} / \mathrm{s}$. However, Fig. 4 exhibits that the total pressure rise $\Delta P_{t}$ is about zero in the vicinity of $\dot{V}^{\prime}=0.01 \mathrm{~m}^{3} / \mathrm{s}$. In other words, the stall phenomenon occurs only in the domain of positive $\Delta P_{t}$. The situation applies to other disk rotating speeds. Therefore, as the first approximation, $\Delta P_{t}=0$ may be considered the criterion for the onset of stall phenomenon in the rotating-disk system.

The power consumption characteristics of the disk assembly is shown in Fig. 7. At the same rotational speed, the power consumed by the fluid $L^{\prime}$ is practically proportional to the volumetric flow rate $\dot{V}^{\prime}$. The slope increases with an increase in $n$. It is also seen that less power is expended when the rotor functions as a fan. The fluid uses up more power in overcoming the resistance in the core at a higher $\dot{V}^{\prime}$.

Figure 8 plots the fan efficiency $\eta$ in the positive $\Delta P_{t}$ region. There is a peak efficiency at each rotational speed. The broken line is the locus of the peak efficiencies. For the rotor core tested, the optimum peak efficiency is approximately $26 \%$ and occurs at $n=16 \mathrm{~Hz}$ and $\dot{V}^{\prime}=$ $0.0035 \mathrm{~m}^{3} / \mathrm{s}$.

In Fig. 9, the dimensionless moment coefficient $C_{m}$ is plotted against the rotational Reynolds number $R e_{w}$ with the radial Reynolds number $R e_{Q}$ as the parameter. The radial Reynolds number is defined as $R e_{Q}=\dot{V}^{\prime} / 2 \pi s v$. The moment coefficients for a free disk in the laminar and turbulent flow regimes (Schlichting 1972) are superimposed in the figure for comparison. At high values of $R e_{Q}$, the moment coefficient $C_{m}$ is higher than that of the free disk and decreases with an increase in $R e_{w}$. When $R e_{Q}$ is below a critical value, the $C_{m}$ curve is concaved with a minimum. The coefficient $C_{m}$ at lower $R e_{w}$ is less than that on a free disk, while it exceeds that on a free disk at higher $R e_{w}$. When all curves are extrapolated rightward (not shown) in Fig. 9, they converge at $R e_{Q}$ of about $6 \times 10^{5}$.

\section{Conclusions}

The pressure drop and torque characteristics of multiple disk assemblies are experimentally investigated. It is concluded from the study that

(i) The rotor core functions as a fan at lower flow rates but becomes a flow resistance in the higher flow regime. 
(ii) At a given rotational speed, the power expended by the fluid is nearly proportional to the through-flow rate. The slope becomes steeper as the rotational speed increases. The fluid consumes less power when the rotor functions as a fan and expends more power when the rotor becomes a flow resistance.

(iii) When the core functions as a fan, there is an operating condition (optimum rotational speed and flow rate) which yields the optimum peak fan efficiency.

(iv) The moment may be greater or lower than that on a free disk depending upon the through-flow rate. When the through-flow rate is below a critical value, the moment curve has a minimum at certain value of the rotational Reynolds number.

(v) The onset of stall propagation may be determined from a plot of $\Delta P_{t}$ versus $\dot{V}^{\prime}$, with the rotational speed as a parameter. The $\dot{V}^{\prime}$ where the $\Delta P_{t}$ falls to zero is the critical value for the onset of stall propagation.

\section{References}

Mochizuki, S.; Tanida, Y. 1979: Unsteady flow phenomena in multiple disk fans. ASME Paper 79-FE-10

Mochizuki, S.; Yang, Wen-Jei; Yagi, Y.; Lleno, M. 1983: Heat transfer mechanisms and performance in multiple parallel disk assemblies. J. Heat Transfer 105, 598-604

Mochizuki, S.; Yang, Wen-Jei 1985: Self-sustained radial oscillating flows between parallel discs. J. Fluid Mech. 154, 377-397

Owens, J. M. 1984: Fluid flow and heat transfer in rotating disc system. In: Heat and mass transfer in rotating machinery (eds. Metzger, D. E.; Afgan, N. H.), pp. 81-103. Washington, DC: Hemisphere

Schlichting, H. 1972: Boundary layer theory, 7th ed. New York: McGraw-Hill

Sim, Y. S.; Yang, Wen-Jei 1984: Numerical study on heat transfer in laminar flow through co-rotating parallel disks. Intern. J. Heat Mass Transfer 27, 1963-1970

Sim, Y. S.; Yang, Wen-Jei 1985: Turbulent heat transfer in corotating annular disks. Numerical Heat Transfer J. (in print)

Received June 11, 1985 